\title{
The Value of CA 125 and CA72-4 in Management of Patients with Epithelial Ovarian Cancer
}

\author{
Salah T. Fayed ${ }^{1, \#}$, Samira M. Ahmad ${ }^{2}$, \\ Samar K. Kassim ${ }^{3}$ and Ali Khalifa ${ }^{3}$ \\ ${ }^{1}$ Departments of Obstetrics and Gynecology, \\ Ain Shams Faculty of Medicine, Cairo, Egypt \\ ${ }^{2}$ Atomic Energy, NCRRT \\ ${ }^{3}$ Department of Biochemistry, Ain Shams \\ Faculty of Medicine, Cairo, Egypt
}

\begin{abstract}
The role of the tumor markers CA125 and CA72-4 has been evaluated in the diagnosis and management of ovarian cancer. Both markers were measured in 30 patients with proven epithelial ovarian cancer, 30 patients with benign pelvic masses and 30 normal women. CA125 and CA72-4 were measured using the luminometric immunoassay and immunoradiometric assay respectively. All patients with ovarian cancer were submitted to surgical staging and cytoreduction followed by adjuvant platinum based chemotherapy for 3-6 courses. Fixing the specificity at $95 \%$, CA125 had a sensitivity of $76.7 \%$ at a cut-off $85 \mathrm{u} / \mathrm{ml}$ while CA72-4 had a sensitivity of $70 \%$ at a cut-off $8.5 \mathrm{u} / \mathrm{ml}$. The combination of CA72-4 with CA125 increased the sensitivity to $95 \%$ while fixing the specificity at $95 \%$. Among seven cases with stage I and II ovarian cancer five cases had CA125 level below $85 \mathrm{U} / \mathrm{ml}$, three patients out of them had CA72-4 above $8.5 \mathrm{U} / \mathrm{ml}$. CA $72-4$ could reflect the residual disease following cytoreduction and could improve the detection of relapse by CA125.

CONCLUSION: CA72-4 could complement the standard tumor marker CA125 both in diagnosis and follow up of patients with epithelial ovarian cancer.
\end{abstract}

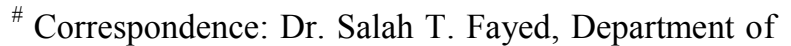
Obstetrics and Gynaecology, Ain Shams University Hospitals, Abbassia, Cairo 11566, Egypt, Fax: +202484 7653, E-mail: ahmed_kassim@usa.net
}

KEYWORDS: CA125, CA72-4, ovarian cancer, tumor markers, optimal cytoreduction, suboptimal cytoreduction

\section{INTRODUCTION}

Despite the advances in diagnostic techniques, cytoreductive surgery and chemotherapy, the overall five year survival of $38 \%$ has not changed over the past three decades [8]. The role of tumor markers in diagnosis and monitoring of ovarian cancer is well established. Since introduced in 1981, CA125 has proved to be a useful first generation tumor marker in ovarian cancer [2]. It has been used to differentiate benign from malignant pelvic masses, to monitor the response to chemotherapy and to detect relapse after initial response to treatment. Unfortunately, CA125 is not elevated above $35 \mathrm{U} / \mathrm{ml}$ in about $50 \%$ of stage I disease especially in the mucinous type [9], while it is elevated in up to $50 \%$ of patients with benign pelvic diseases [10]. Moreover, over $50 \%$ of patients undergoing second look laparotomy will have residual (mostly minimal) disease in spite of their normal serum CA125 level [5]. In order to enhance the sensitivity and specificity of CA 125 , different panels of tumor markers have been tried. Among the studied markers is CA72-4, a high molecular weight glycoprotein first described by Colcher in 1981 [4]. This marker was found to be elevated in a variety of adenocarcinomas including gastric, colon, breast and ovarian adenocarcinoma especially the mucinous sub-type [3]. Used alone or in combination with CA125, CA72-4 proved to be useful marker in ovarian cancer [12]. In this 
study we have evaluated the combination of CA125 at a cut-off $85 \mathrm{U} / \mathrm{ml}$ and CA72-4 at a cutoff $8.5 \mathrm{U} / \mathrm{ml}$ in the primary diagnosis of ovarian cancer. Lower cut-offs $(35 \mathrm{U} / \mathrm{ml}$, and $3 \mathrm{U} / \mathrm{ml}$ respectively) were used to monitor the course of the disease and to detect relapse after initial response to chemotherapy.

\section{SUBJECTS AND METHODS}

This study was conducted in the period from March, 1994 through April, 1996. It included 30 patients with epithelial ovarian cancer, their ages ranged between (19-65), 30 patients with benign pelvic diseases their ages ranged between $(20$ 60 ) and 30 normal women with no past or family history of ovarian or breast cancer their ages ranged between (22-63). All patients were submitted to complete physical examination, pelviabdominal ultrasonography. Computed tomography was ordered on an individual basis. Blood samples were withdrawn from all patients, serum separated, frozen and stored at $-70{ }^{\circ} \mathrm{C}$ for later assay of the tumor markers CA125 and CA72-4. All patients with ovarian cancer were submitted to surgical staging and cytoreduction including total abdominal hysterectomy and bilateral salpingoopharectomy, omentectomy, appendectomy, selective pelvic and para-aortic lymphadenectomy, peritoneal cytology and removal of all resectable tumor masses. Peritoneal cytology was performed in the absence of gross peritoneal pathology. Histopathological examination was undertaken for final diagnosis, accurate staging, degree of differentiation and histological subtype. Further blood samples were withdrawn from patients with ovarian cancer at one, six, twelve and eighteen month postoperatively. Except for patients with stage I a disease, all patients received platinum based combination chemotherapy every 3-4 weeks for 3-6 courses. Quantitative assay of CA125 using the luminometric immunoassay was performed [15]. The kit was supplied by BYK-Sangtic Diagnostic (Germany). The immunoradiometric assay for the quantitative determination of TAG 72 (tumor associated glycoprotein 72) was used for determination of CA72-4 [13]. Response to treatment and performance of patients were judged through regular clinical assessment, imaging techniques and tumor marker assay.

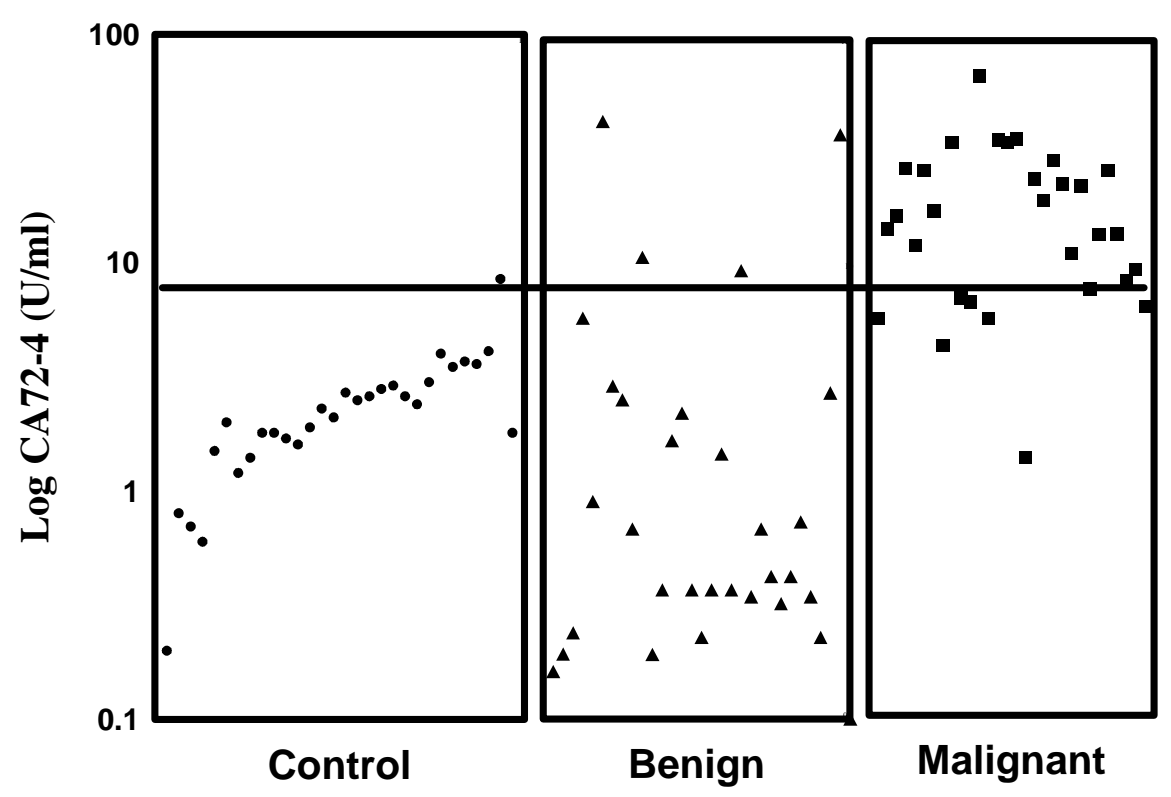

Fig. 1. Serum CA72-4 in control, benign, and ovarian cancer patients. 
Table 1

The sensitivity of CA125 and CA $72-4$ at $95 \%$ fixed specificity.

\begin{tabular}{cc}
\hline Marker & Sensitivity \\
\hline CA125 $(85 \mathrm{u} / \mathrm{ml})$ & $76.7 \%$ \\
CA72-4 $(8.5 \mathrm{u} / \mathrm{ml})$ & $70 \%$ \\
CA125 + CA72-4 & $95 \%$ \\
\hline
\end{tabular}

\section{RESULTS}

Among the studied cases of ovarian cancer, only 7 out of 30 patients were stage I and II disease $(23 \%)$ while the rest of them were stage III and IV (77\%). Mucinous histological subtype was diagnosed in 12 patients $(40 \%)$ while nonmucinous tumors were diagnosed in $(60 \%)$. Poorly differentiated carcinoma (grade 3 ) was present in $36 \%$ while moderately differentiated (grade 2) and mixed grade 1 and 2 was present in $64 \%$ of cases.

Fixing the specificity at $95 \%$ for both control and benign groups, the cut-off for CA125 was $85 \mathrm{U} / \mathrm{ml}$ and was $8.5 \mathrm{U} / \mathrm{ml}$ for CA72-4 (Figure 1). At these cut-offs, the sensitivity of CA125 was $76.7 \%$ and for CA72-4 it was $70 \%$. When applied simultaneously the sensitivity increased to $95 \%$ at the same specificity (Table 1).

Based on the histological subtype, among 12 cases with mucinous cystadenocarcinoma CA125 was elevated above $85 \mathrm{U} / \mathrm{ml}$ in 7 cases $(58 \%)$ while 10 patients out of 12 had CA72-4 above $8.5 \mathrm{U} / \mathrm{ml}(83 \%)$. On the other hand serum CA125 exceeded the cut-off in all patients with non-mucinous variety of ovarian cancer $(100 \%)$ while only 13 out of 18 cases had elevated CA724 above $8.5 \mathrm{U} / \mathrm{ml}(72 \%)$. When the tumor stage was considered, only 2 out of 7 patients with stage I and II disease had CA125 above $85 \mathrm{U} / \mathrm{ml}$ (28\%) while 5 patients had CA72-4 above $8.5 \mathrm{U} / \mathrm{ml}(71 \%)$ and 2 cases had non of these markers elevated.

Although maximal surgical effort was performed, optimal cytoreduction was possible only in $57 \%$ of patients with different stages of ovarian cancer. The rest were suboptimally cytoreduced (43\%). Among 11 patients with preoperative elevation of CA72-4 who underwent optimal cytoreduction, 9 patients $(85 \%)$ had the tumor marker level below $8.5 \mathrm{U} / \mathrm{ml}$ one month
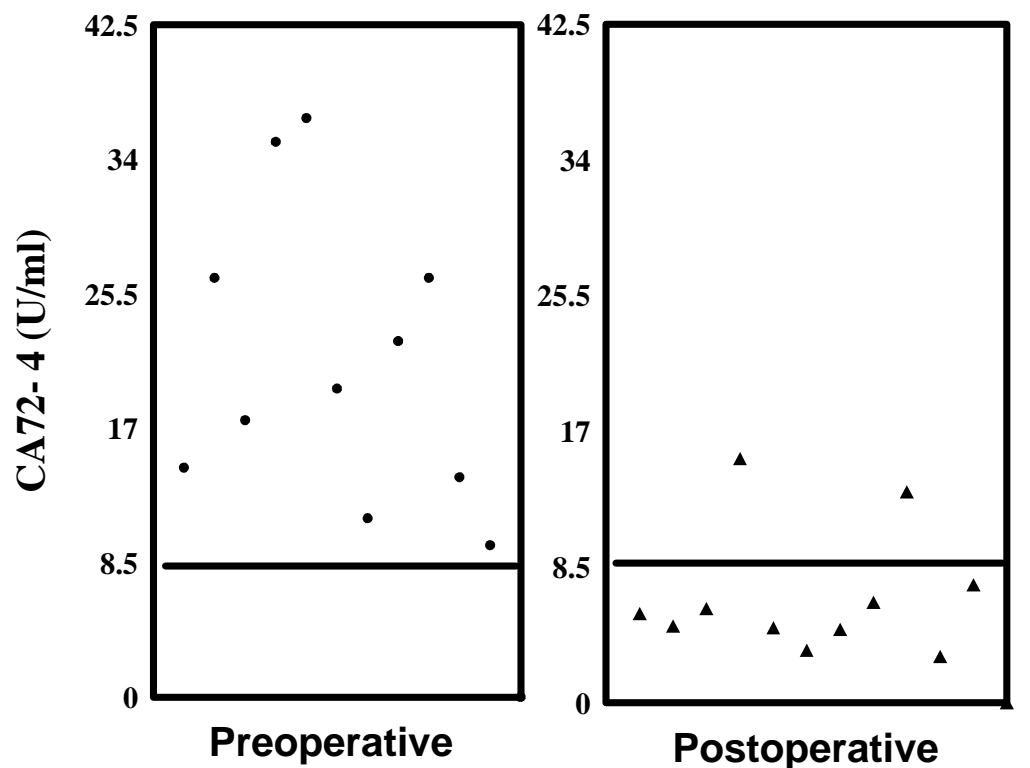

Fig. 2. Serum CA72.4 before and one month after optimal cytoreduction. 
Table 2

Mean values of CA125 and CA72-4 one month postoperative

\begin{tabular}{ccccl}
\hline Marker & $\begin{array}{c}\text { Optimal cytoreduction } \\
(\mathrm{n}=17)\end{array}$ & $\begin{array}{c}\text { Suboptimal cytoreduction } \\
(\mathrm{n}=13)\end{array}$ & $\mathrm{t}$ & $\mathrm{P}$ \\
\hline CA 125 & $74.4 \pm 32.4$ & $86.3 \pm 49.3$ & 0.62 & 0.54 \\
CA 72-4 & $5.1 \pm 3.3$ & $10.2 \pm 9.2$ & 1.77 & 0.104 \\
\hline
\end{tabular}
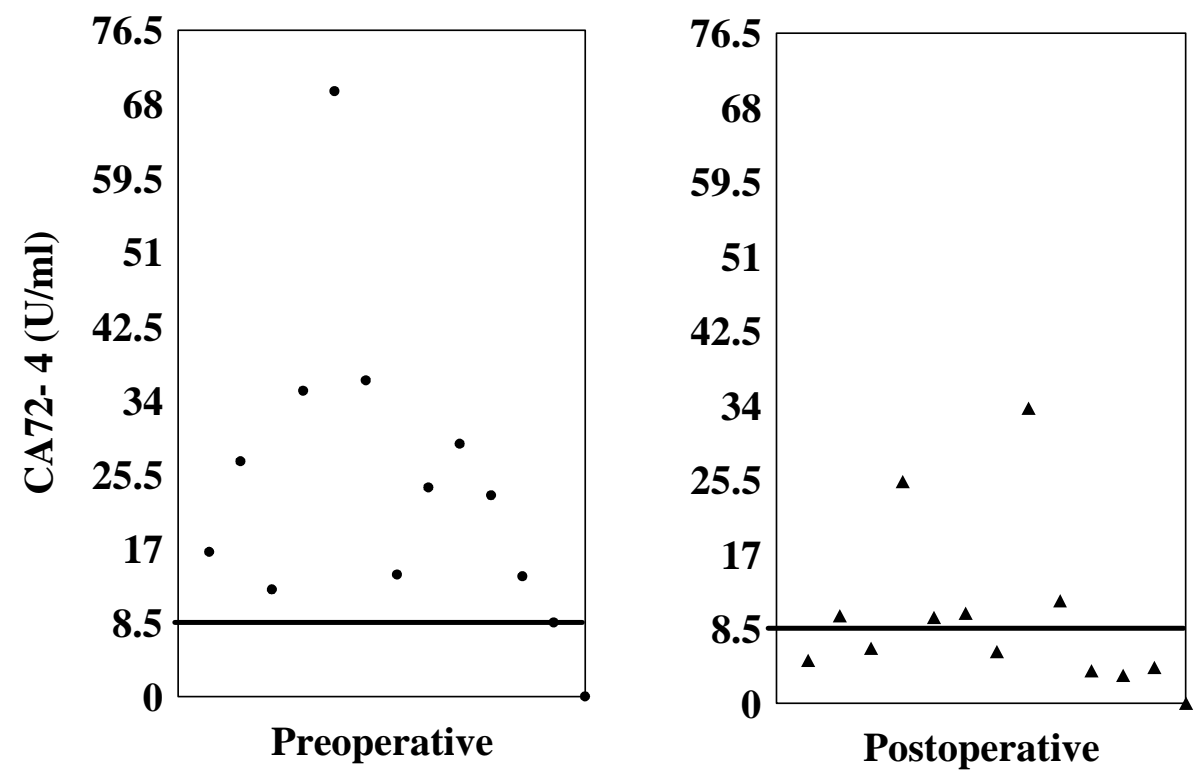

Fig. 3. CA72-4 before and one month after suboptimal cytoreduction.

postoperatively (Figure 2), while only 6 out of 12 patients who underwent suboptimal cytoreduction had tumor marker level below $8.5 \mathrm{U} / \mathrm{ml}$ $(50 \%)$ (Figure 3). Similar testing of CA125 revealed normal level below $85 \mathrm{U} / \mathrm{ml}$ only in 7 out of 11 patients who were optimally cytoreduced $(63 \%)$ while 5 out of 11 patient with suboptimal cytoreduction fell below $85 \mathrm{U} / \mathrm{ml}$ (45\%) (Table 2).

Used to monitor the course of the disease, CA125 was elevated above $35 \mathrm{U} / \mathrm{ml}$ in 4 out of 6 patients with proven relapse after initial response to treatment. On the other hand, CA72-4 was elevated above $3 \mathrm{U} / \mathrm{ml}$ in 5 out of the 6 patients with relapse including the 2 cases with false negative CA125. After 18 month of follow up 11 patients out of 30 were alive with no evidence of disease either clinically or by imaging techniques. Ten out of these 11 cases had CA125 below $35 \mathrm{U} / \mathrm{ml}$. Only 5 of these patients had CA72-4 below $3 \mathrm{U} / \mathrm{ml}$ (Figure 4).

\section{DISCUSSION}

The role of tumor markers in diagnosis and monitoring of ovarian cancer is well established. CA125 is by far the most commonly used marker and the most useful in reflecting the disease status especially in non-mucinous ovarian cancer [10]. The strategy of concomitant assay of different tumor markers to enhance sensitivity of different markers is well known [6]. The present study tests the possible gain from adding CA72-4 

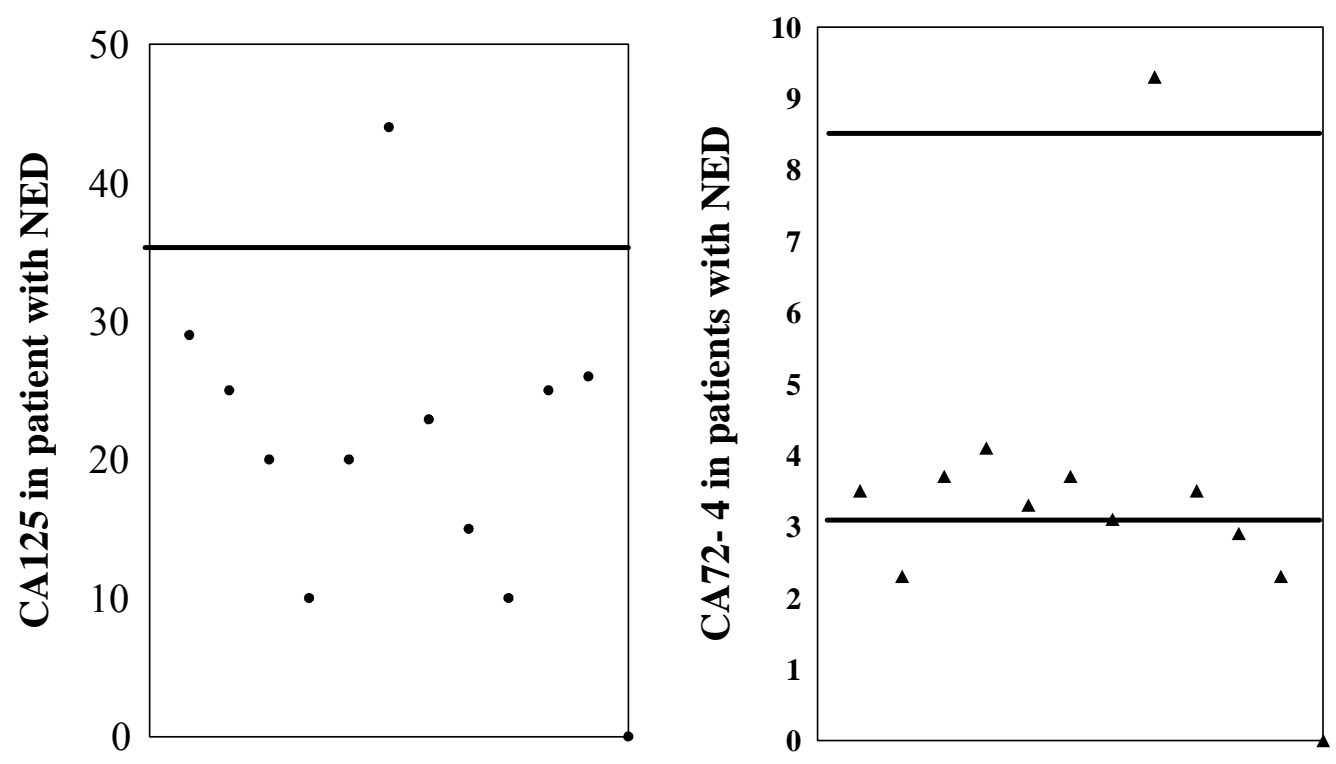

Fig. 4. CA125 and CA72-4 in patients with no clinical or radiological evidence of disease after 18 months.

to CA125 in the management of patients with ovarian cancer. We have confirmed the previous finding that CA72-4 is the leading marker in mucinous cystadenocarcinoma while CA125 is the leading marker in non-mucinous carcinoma [7]. In our study the mucinous histological variety is over represented $(40 \%)$ instead of the normal distribution (15\%). Among patients with stage I and II disease CA72-4 was more frequently elevated than CA125 (5/7 versus $2 / 7$ ). This low detection rate of CA125 may be in part due to the higher cut-off used and the tendency of mucinous subtype to present at earlier stages [1]. When both markers were assayed at one month postoperatively, $9 / 11$ patients had CA72-4 below $8.5 \mathrm{U} / \mathrm{ml}$ after optimal cytoreduction while only 6/12 with suboptimal cytoreduction fell below this cut-off. On the other hand 7/11 patients with optimal cytoreduction and 5/11 patients with suboptimal cytoreduction who had preoperative elevation of CA125 were below the mentioned cut-off. This apparent advantage of CA72-4 over CA125 in reflecting the residual tumor burden needs confirmation by a larger study. Moreover this could be in part due to the greater prevalence of the mucinous cancers among patients with earlier stages (5/7 in this study) when optimal cytoreduction is more feasible. As second look surgery was not performed to judge the response to treatment, patients with no clinical or radiographic evidence of disease were considered free of cancer at 18 month. These techniques are of limited sensitivity and could miss tumor masses less than $2 \mathrm{~cm}$ [14]. Among 11 patients apparently with NED, only one patient (1/11) had CA125 above $35 \mathrm{U} / \mathrm{ml}$, while 5 patients $(5 / 11)$ had CA72-4 above $3 \mathrm{U} / \mathrm{ml}$. It's worth noting that the percentage of patients with positive CA72-4 nearly equals the expected false negative percentage of $50 \%$ for CA125 at the time of second look surgery [5]. Meier et al. [11] had found $6 / 27$ patients with true positive elevation of CA72-4 although they had negative CA125 $(<35 \mathrm{U} / \mathrm{ml})$ at the time of second look laparotomy. Whether CA72-4 could predict the presence of minimal residual disease with normal CA125 is a postulate that calls for further confirmation. Along the follow up period, six patients had overt relapse after initial response to treatment. CA125 was elevated above $35 \mathrm{U} / \mathrm{ml}$ in $4 / 6$, while $5 / 6$ patients had elevated CA72-4 above $3 \mathrm{U} / \mathrm{ml}$. All patients with proven relapse had one or both markers elevated. 


\section{CONCLUSION}

CA72-4 is a useful tumor marker that can complement CA125 especially in mucinous ovarian adenocarcinoma. Its advantage over CA125 in detection of minimal residual disease at the time of second look needs to be confirmed by a larger, second look based study.

\section{References}

[1] Aure, J.C., Hoeg, K. and Kolstad P. Clinical and histological studies of ovarian carcinoma: longterm follow up of 990 cases. Obstet. Gynecol. 37(1), (1971).

[2] Bast, R.C., Feeney, M., Lazarus, H., Nadlar, L.M., Colvin, R.B. and Knapp R.C. Reactivity of a monoclonal antibody with human ovarian carcinoma. J. Clin. Invet. 68, (1981) 1331-1337.

[3] Bayerl, B., Meier, W., Steiber, P., Albiez, M., Eirmann, W. and Fateh-Moghadam, A. Significance of CA72-4 in the diagnosis of ovarian cancer. In: Recent results in tumor therapy and diagnosis. Klapdor, R. (ed) Zuckschwedt-Verlag, Munich, (1990) 111-112.

[4] Colcher, D., Horan Hand, P., Nuti, M. and Schlom, J.A. Proc. Nat. Acad. Sci. 78, (1990) 3199.

[5] DiSaia, P.J. and Creasman, W.T. (ed) Epithelial ovarian cancer. In: Clinical Gynecologic Oncology. Mosby Year Book, St. Louis, Missouri, USA (1997) 326.

[6] Fioretti, P., Gadducci, A., Ferdeghini, M., Prontera, C., Malagnino, G., Facchini, V., Mariani, G. and Bianchi, R. The concomitant determination of different serum tumor markers in epithelial ovarian cancer: relevance for monitoring the response to chemotherapy and follow up of patients. Gynecol. Oncol. 44(2), (1992) 155-156.

[7] Hasholzner, U., Baumgartner, L., Stieber, P.,
Meier, W., Reiter, W., Pahl, H. and FatehMoghadam, A. Clinical significance of the tumor markers CA125 II and CA72-4 in ovarian carcinoma. Int. J. Cancer (pred Oncol) 69, (1996) 329-334.

[8] Hoskins, W.J., Perez, C.A. and Young, R.C. (ed) Epidemiology of gynaecologic cancer. In: Principles and Practice of Gynecologic Oncology. Lippincott Company, Philadelphia, Pennsylvania, USA (1992) 326.

[9] Jacobs, I. and Bast, R.C. The CA125 tumor associated antigen: a review of the literature. Human Reprod. 4, (1989) 1-12.

[10] Kenemans, P., Yedema, C.A., Bon, G.G. and von Mensdorff-Pouilly, S. CA125 in gynecological pathology- a review. Eur. J. Obstet. Gynecol. and Reprod. Biol. 49, (1993) 115-124.

[11] Meier, W., Bayerl, B., Steiber, P., Eiermann, A. and Fateh-Mohadam, A. Serum levels of CA125 and CA72-4 at the time of second look laparotomy in ovarian cancer patients. Geburtsh. Frauenheilk. 48, (1988) 331.

[12] Negishi, Y., Iwabuchi, H., Sakunaga, H., Sakamoto, M., Okabe, K., Sato, H. and Asano, G. Serum and tissue measurements of CA72-4 in ovarian cancer patients. Gynaecologic Oncology 48, (1993) 148-154.

[13] Ohuchi, N., Gero, E., Mori, S., Akimoto, M., Matoba, N., Nishihira, T., Hirayama, K., Colcher, D. and Schlom, J. Clinical evaluation of CA72-4 immunoradiometric assay for serum TAG-72 antigen in patients with carcinoma. J. Tumor Mark Oncol. 5(1), (1990) 1-10.

[14] Piver, M.S., (ed) Ovarian epithelial cancer. In: Handbook of gynaecologic oncology, A. Little, Brown and Company, Boston, Massachusetts, (1996) 22.

[15] Wood, W.G. Automation of luminescence immunoassays with coated tubes using the LIA MAT 300 system as example. In: Tumor Diagnosis and therapy. Klapdor, R. (ed) W. Zuckschwerdt Verlag, München, (1992) 348350 . 


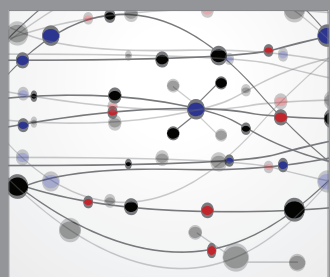

The Scientific World Journal
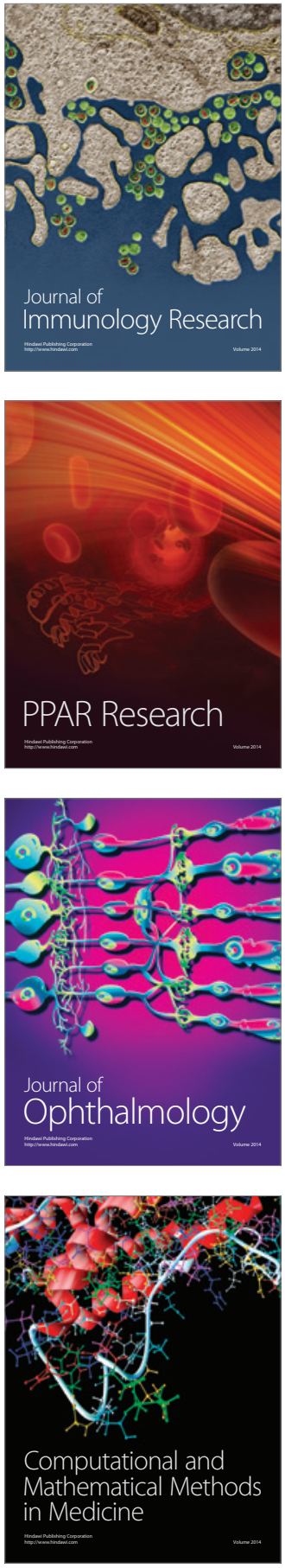

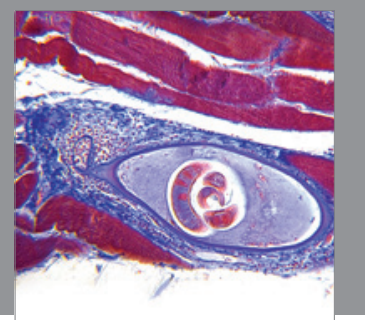

Gastroenterology

Research and Practice
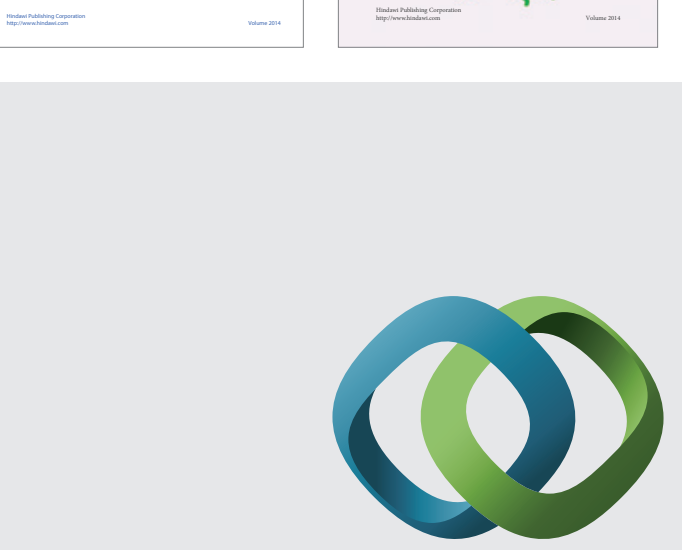

\section{Hindawi}

Submit your manuscripts at

http://www.hindawi.com
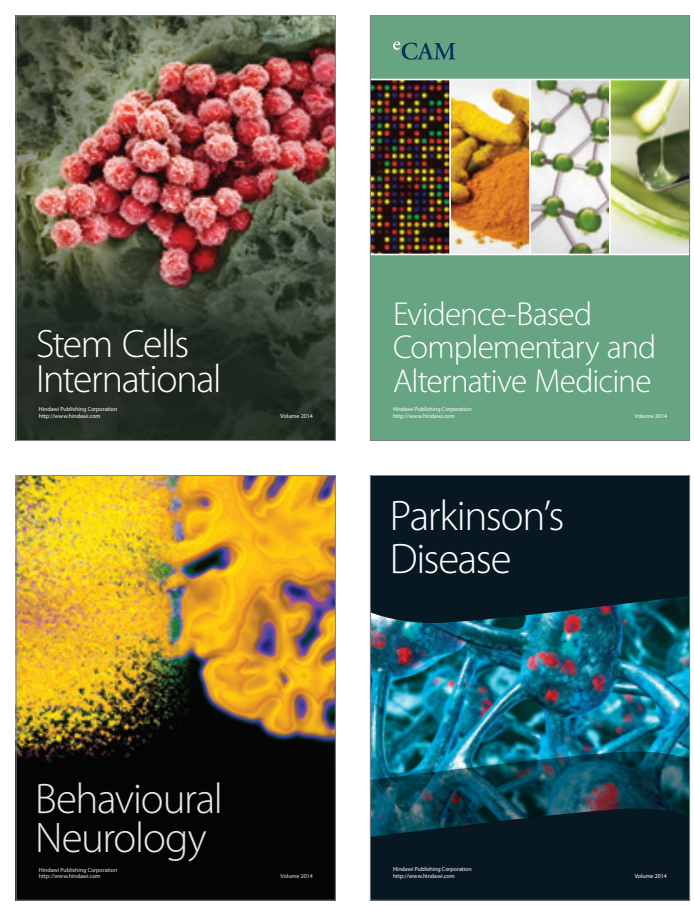

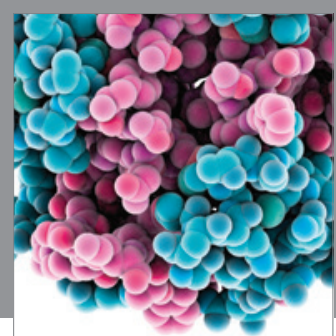

Journal of
Diabetes Research

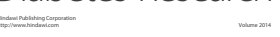

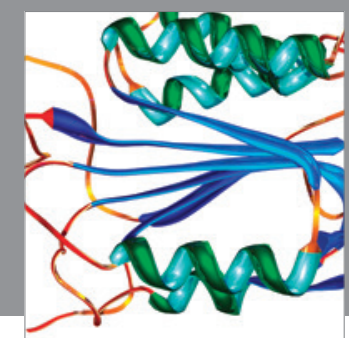

Disease Markers
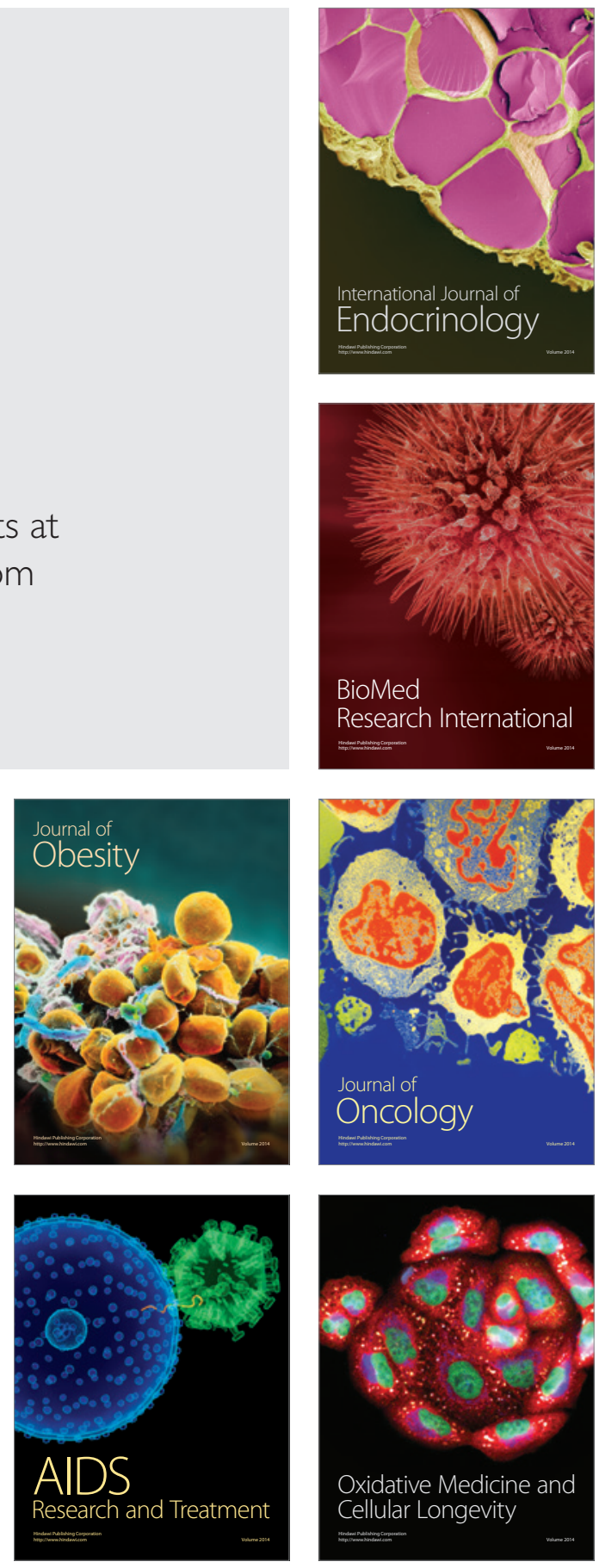